\title{
Odontomes involving both the jaws: A case report
}

\author{
Jaya Singhvi', Arushi Pandey ${ }^{2, *}$, Gurkiran Kaur ${ }^{3}$ \\ ${ }^{1}$ Private Practitioner, ${ }^{2,3}$ Professor and HOD, ${ }^{1}$ Dept. of Orthodontics, ${ }^{2}$ Dept. of Oral Pathology, ${ }^{1}$ Kota, Rajasthan, ${ }^{2}$ College of \\ Dental Sciences, Rau, Indore, Madhya Pradesh, ${ }^{3}$ PDM Dental College \& Research Institute, Bahadurgarh, Haryana, India
}

*Corresponding Author:

Email: roshipandey@yahoo.co.in

\begin{abstract}
Introduction: Odontomes are most common type of odontogenic tumors. They are generally asymptomatic and discovered on routine radiographic examinations. Morphological classification of odontomes can be doneas complex and compound odontomes. Here we document a case of a 15 year old boy who attended our dental department with apparent malocclusions. A panoramic radiograph revealed presence of multiple radio opaque masses in maxillary as well as mandibular arches. These structures were seen associated with impacted permanent teeth. The histopathological examination confirmed the diagnosis of them as being complex odontomes.
\end{abstract}

Keywords: Odontogenic tumor, Odontome, Complex, Compound.

\section{Introduction}

In 1937 it was Sprawson who did a thorough review on odontomes, where he mentioned that Paul in 1867 used the term "odontoma" to describe the set of odontogenic tumors whose etiology was due to the overgrowth of transitory or complete odontogenic tissue. ${ }^{1}$ Morphologically odontomes are classified into two groups as compound and complex odontoma. Compound odontoma represents dental tissues having orderly distribution with many miniature tooth like structures whereas in complex odontomes no such tooth like resemblance is seen of the histologically evident dental tissues. Clinically, three variants are recognized which include central, peripheral and erupted. Central (intraosseous) are confined to the bone, peripheral (extraosseous) are found in the oral mucosa covering the tooth bearing areas of maxilla and mandible, while erupted are essentially central odontomes which erupt out into the oral cavity. ${ }^{2}$

Odontomes are generally associated with pathologic changes such as malformations, impactions, delayed eruptions, malpositioning, cyst formation or displacement and resorption of adjacent teeth. Rarely, they are seen associated with absence of one or more teeth. $^{3}$

Literature states that odontomes are the most common odontogenic tumors. Males and females are equally affected. There is an increased prevalence in children and adolescents. Here, we report a unique case of multiple odontomes, involving both maxillary and mandibular arches causing impaction of several permanent teeth and retention of deciduous teeth. ${ }^{4}$

\section{Case Report}

A 15-year-old boy reported to the dental OPD with doubts about the delay in exfoliation of deciduous teeth and malalignment of permanent teeth. Intraoral examination revealed spacing in between teeth and that multiple deciduous teeth were retained and permanent teeth were missing. Also, expansion of alveolar bone was evident below the retained deciduous teeth. To confirm the eruptive status/root formation a panoramic radiograph was taken which revealed multiple radiopaque masses in the maxillary and mandibular anterior region with multiple impacted teeth (maxilla 21, 23 and mandible 32, 43) (Fig. 1). To pinpoint the exact location and the number of radiopaque masses, computerized tomography was advised, which exhibited multiple impacted masses labial and lingual to the impacted permanent teeth. For treatment multidisciplinary approach was planned. The treatment approach was first to remove the radiopaque masses and expose the crown of favorably impacted teeth for aligning in the arch. Under local anesthesia the retained deciduous teeth $(53,61,73,83,84)$ were extracted first in the maxillary arch followed by the mandibular arch. The impacted teeth were then attached with an orthodontic device for traction (Fig. 2, 3).

Specimens were sent for histopathological examination. Soft tissue examination revealed a nonkeratinized, stratified squamous epithelium overlying a vast expanse of connective tissue similar to the primitive mesenchyme. The stroma was abundant cell rich and loose, composed of delicate interlacing network of collagen fibres, plump fibroblasts, fibrocytes and a large number of nests of odontogenic rests which were suggestive of dental follicle.

The decalcification of the hard tissues which was carried out in $8 \%$ potassium formate formic acid, and then stained with hematoxylin and eosin revealed large amounts of irregularly deposited mature tubular dentin, enclosing clefts and empty circular spaces representing the places of enamel deposition. Adjacent with the masses of dentin was the connective tissue resembling normal pulp comprising of delicate network of collagen fibres, fibroblasts, fibrocytes, few inflammatory cells 
and blood vessels. Aggregate of cementum were also seen (Fig. 4a and b).

Correlating with the clinical and radiographic picture, the histological description of haphazard deposition of dental tissue was most consistent with the diagnosis of complex composite odontome.

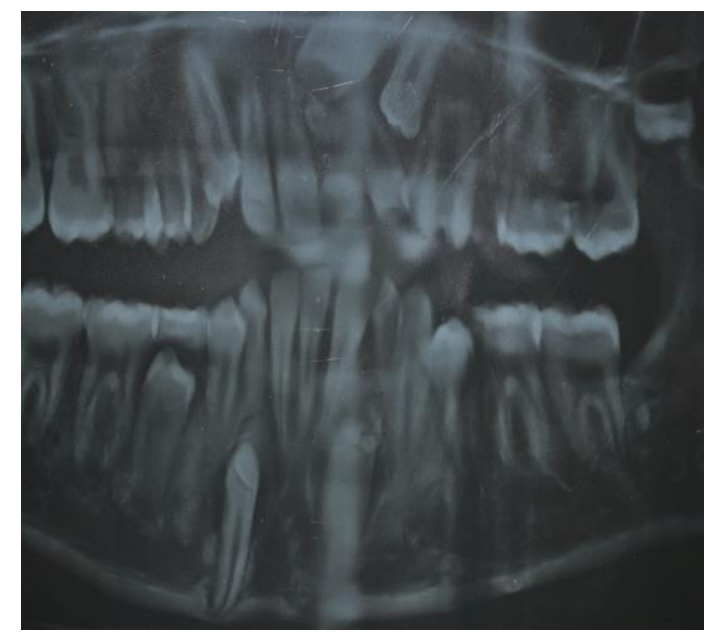

Fig. 1: OPG shows multiple impacted teeth

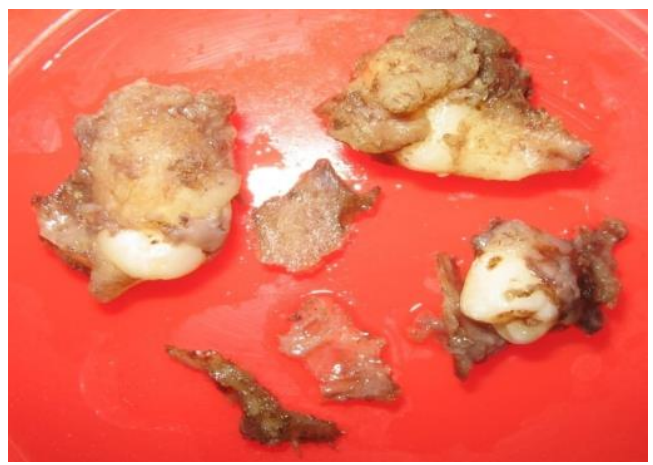

Fig. 2: Gross specimen

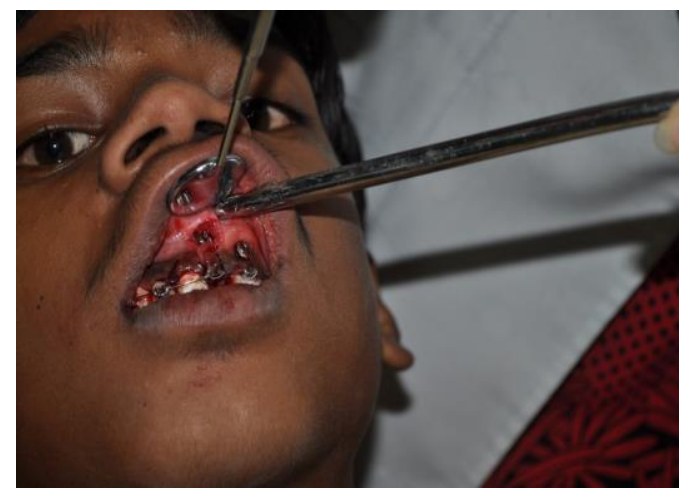

Fig. 3: Orthodontic traction

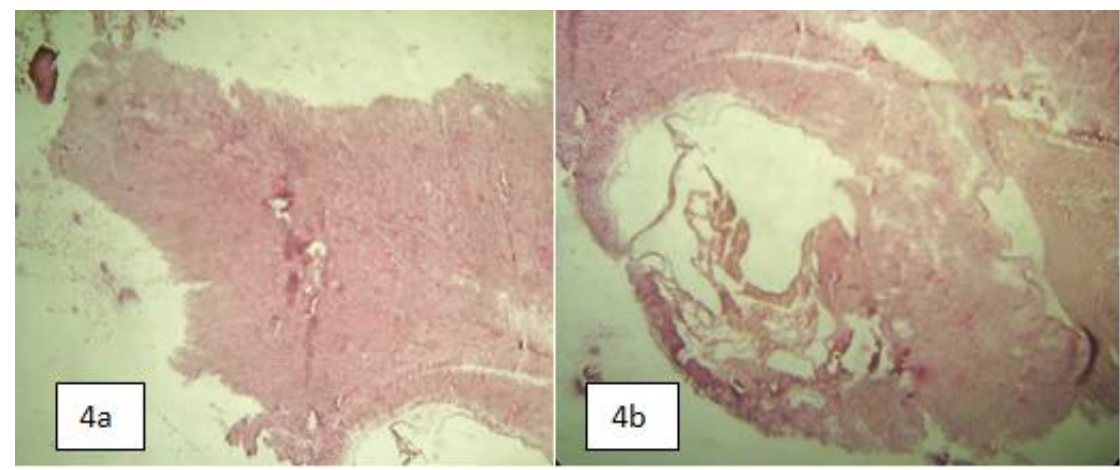

Fig. 4 a and b: Decalcified section showing tubular pattern of dentin with empty spaces of enamel lost during decalcification and fine fibrillary tissue of pulp

\section{Discussion}

Paul Broca was the first to use the term "odontoma" in 1867. Thoma and Goldman described five subtypes of odontoma based on histomorphological features. These included the geminated composite odontomes, compound composite odontomes, complex composite odontomes, dilated odontomes and cystic odontomes. $^{5}$

According to the latest classification, WHO has placed odontomas under the heading of odontogenic tumors of odontogenic epithelium with odontogenic mesenchyme with or without hard tissue formation along with ameloblastic fibro-odontoma which is regarded as an immature precursor of complex odontoma.
The formation of odontomes is unknown but numerous etiologies have been proposed such as: trauma during primary dentition, or as a result of inflammatory and infectious processes, hereditary anomalies (Gardeners syndrome, Hermann's syndrome), odontoblastic hyperactivity or alteration of the genetic components responsible for controlling dental development. ${ }^{6}$ Gurkiran et al. suggested that a mutation in the dental lamina or of the tooth germ itself may change the inherent capacity of odontogenic epithelium to go through the cap and bell stages necessary for tooth formation and yet retain its ability to stimulate mesenchymal differentiation necessary for dentine formation and to form functional ameloblasts that form enamel leading to the development of a composite odontoma. They elaborated that the genes present in normal primordia of tooth were found in the 
odontogenic tumor epithelium. A possible explanation for this is that the epithelial cells of the odontogenic tumor are recapitulating genetic programs expressed during normal odontogenesis, but with abnormal expression of genes. ${ }^{7}$

The average age of presentation of erupted odontoma is in the second decade of life as was with the present case. A study carried out to find any relation between the patient's age and the variety of odomtoma suggested that the compound odontomas are found more in younger individuals in the anterior region, whereas complex odontomas occurred in older age group with molar region being the site of occurrence in them. ${ }^{8}$ In our case the odontomes were present in the anterior region of both the jaws. Frequently, odontomas occur in the place of missing teeth or alternatively if all the teeth are present an odontoma may represent a supernumerary tooth. Over retained primary teeth and/or unerupted permanent teeth often lead to the identification of these lesions ${ }^{9}$ as seen in this case. Radiographically, they appear predominantly radiopaque and surrounded by a thin well circumscribed radiolucent band. Odontoma is often associated with an unerupted tooth. The present case had a similar radiographic picture with the size of the lesion larger than usual. However, ameloblastic fibroodontoma or Odontoameloblastoma has to be considered in the differential diagnosis and has to be checked by histologically if the lesion is located pericoronally. ${ }^{10}$

\section{Conclusion}

The management of odontomas associated with an impacted tooth comprise of surgical removal of the lesion along with orthodontically aligning the impacted tooth. It has been reported that such impacted teeth tend to erupt, regardless of the amount of root formation. Extraction of such impacted teeth is suggested only in those cases where the tooth is abnormally oriented, morphologically changed or present with cystic changes.

\section{Conflict of Interest: None.}

\section{References}

1. Philipsen HP, Reichart PA, Praetorius F. Mixed odontogenic tumours and odontomas. Considerations on interrelationship. Review of the literature and presentation of 134 new cases of odontomas. Oral Oncol 1997;33:86-9.

2. Neville BW, Damm DD, Allen CM, Bouquot JE. Oral and Maxillofacial Pathology 2nd ed. Philadelphia: WB Saunders Co; 2005.p. 631-32.

3. Gomel M, Seçkin T. An erupted odontoma: case report. $J$ Oral Maxillofac Surg. 1989;47:999-1000.

4. Junquera L, de Vicente JC, Roig P, Olay S, RodriguezRecio O. Intraosseous odontoma erupted into the oral cavity: An unusual pathology. Med Oral Patol Oral Cis Buccal 2005;10:248-51.

5. Cawson RA, Binnie WH, Speight $\mathrm{P}$, Barrett AW, Wright JM. Luca's Pathology of Tumors of the Oral Tissues $5^{\text {th }}$ ed. London: Churchill Livingstone; 1998. p.83-85.

6. Sprawson E. Odontomes. Br Dent J 1937;62:177-201.

7. Kaur GA, Sivapathasundharam B, Berkovitz BK, Radhakrishnan RA. Indian J Dent Res 2012;23:699.

8. Ragalli CC, Ferreria JL, Blasco F. Large erupting complex odontoma. Int J Oral Maxillofac Surg 2000;29:373-74.

9. Ide F, Shimoyama T, Horie N. Gingival peripheral odontoma in an adult: case report. J Periodontol 2000;71:830-32.

11. Thoma, Kurt H. Goldman HM, Gorlin RJ. Thoma's Oral pathology / edited by Robert J. Gorlin, Henry M. Goldman. St. Louis: Mosby, 1970.

How to cite the article: Singhvi J, Pandey A, Kaur G. Odontomes involving both the jaws: A case report. J Dent Specialities 2018;6(2):174-176. 